\title{
Consumo Alimentar e Perfil Antropométrico de Tenistas Amadores e Profissionais
}

\author{
Food Intake and Anthropometric Profile of Amateur and \\ Professionals Tennis Players
}

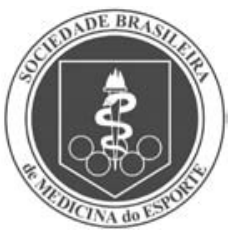

Artigo Original

\author{
Rodrigo Vitasovic Gomes' \\ Sandra Maria L. Ribeiro ${ }^{2}$ \\ Renata Furlan Veibig ${ }^{3}$ \\ Marcelo Saldanha Aoki ${ }^{1,2}$

\begin{abstract}
1. Escola de Educação Física e Esporte da Universidade de São Paulo, São Paulo, Brasil.

2. Escola de Artes, Ciências e Humanidades da Universidade de São Paulo, São Paulo, Brasil. 3. Centro Universitário São Camilo, São Paulo, Brasil.
\end{abstract}

\section{Endereço para correspondência: Prof. Dr. Marcelo Saldanha Aoki Grupo de Pesquisa em Adaptações Biológicas ao Exercício Físico Escola de Artes, Ciências e Humanidades - USP Av. Arlindo Bettio, 1.000 - 03828-000 - São Paulo, SP - Brasil. E-mail: saldanha.caf@usp.br}

Submetido em: 21/11/2008 Versão final recebida em: 10/05/2009 Aceito em: 16/05/2009

\begin{abstract}
RESUMO
Introdução: O perfil antropométrico e o consumo alimentar exercem grande influência no desempenho esportivo; entretanto, essas informações sobre tenistas brasileiros ainda são escassas. Objetivo: Descrever e comparar o consumo alimentar e o perfil antropométrico de tenistas amadores (AM) e profissionais (PRO). Métodos: Foram avaliados 24 tenistas, com a seguinte distribuição: profissionais ( $P R O ; n=9)$ e amadores ( $A M$; $n=15$ ). Os atletas foram avaliados quanto a variáveis antropométricas (peso, estatura, circunferências e dobras cutâneas) e a composição corporal foi estimada por três diferentes protocolos. As dietas foram avaliadas a partir de três diários alimentares. Resultados: Não houve diferença significativa no perfil antropométrico dos atletas PRO em relação aos AM (peso: 69,5 \pm 9,8kg e 66,0 \pm 5,0kg; estatura: 177,9 \pm 4,3cm e 175,6 \pm 2,7cm, IMC: 23,5 $\pm 1,4 \mathrm{~kg} / \mathrm{m}^{2}$ e $22,6 \pm 0,8 \mathrm{~kg} / \mathrm{m}^{2}$ e gordura corporal: $13,0 \pm 5,5 \%$ e 13,7 $\pm 2,4 \%$, respectivamente). Observou-se significativo déficit energético entre a estimativa da necessidade energética e a ingestão energética relatada. O consumo de carboidratos apresentou-se no limite mínimo sugerido (AM: 6,3 \pm 0,5g/kg/dia e PRO: $6,5 \pm 0,7 \mathrm{~g} / \mathrm{kg} / \mathrm{dia})$, enquanto a ingestão de proteínas mostrou-se superior às recomendações disponíveis na literatura (AM: 2,4 \pm 0,2g/kg/dia e PRO: 2,3 \pm 0,3g/kg/dia). Com relação aos minerais, a principal preocupação foi a baixa ingestão de cálcio (AM: 798,1 \pm 786,3mg/dia e PRO: 766,9 $\pm 602,4 \mathrm{mg} /$ dia). Conclusão: Não foram observadas diferenças significativas no perfil antropométrico e no consumo alimentar entre os atletas AM e PRO. Os desvios observados no consumo alimentar reforçam a necessidade de orientação/planejamento nutricional, a fim de atender às demandas específicas da modalidade, visando maximizar o desempenho.
\end{abstract}

Palavras-chave: tênis, dieta, perfil antropométrico.

\section{ABSTRACT}

The anthropometric profile and food intake play a key role in sports performance; however, there is little information available regarding Brazilian tennis players. Aim: the present study aimed to evaluate the food intake and the anthropometric profile of professional and amateur tennis players. Methods: twenty-four tennis players were distributed in two groups: professionals ( $P R O ; n=9)$ and amateurs $(A M ; n=15)$. The athletes were evaluated on their anthropometric measurements (body weight, height, circumferences and skin folders). Body fat was estimated from three different equations. Food intake was determined by a 3-day food diary. Results: there were no significant differences from anthropometric profile between PRO and AM (body weight: $69.5 \pm 9.8 \mathrm{~kg}$ and $66.0 \pm 5.0 \mathrm{~kg}$; height: $177.9 \pm 4.3 \mathrm{~cm}$ and $175.6 \pm 2.7 \mathrm{~cm}$, BMl: $23.5 \pm 1.4$ $\mathrm{kg} / \mathrm{m}^{2}$ and $22.6 \pm 0.8 \mathrm{~kg} / \mathrm{m}^{2}$ and body fat: $13.0 \pm 5.5 \%$ and $13.7 \pm 2.4 \%$, respectively). Significant difference between the energy expenditure and estimation and reported energy intake was observed. Both groups showed low carbohydrate (AM: $6.3 \pm 0.5 \mathrm{~g} / \mathrm{kg} /$ day and PRO: $6.5 \pm 0.7 \mathrm{~g} / \mathrm{kg} /$ day) and high protein intake AM: $2.4 \pm 0.2 \mathrm{~g} / \mathrm{kg} /$ day and PRO: $2.3 \pm 0.3 \mathrm{~g} / \mathrm{kg} /$ day) compared to the current recommendations. Very low calcium intake was observed (AM: $798.1 \pm 786.3 \mathrm{mg} /$ day and PRO: $766.9 \pm 602.4 \mathrm{mg} /$ day). Conclusion: no significant differences were detected for food intake pattern and anthropometric profile between PRO and AM. The results presented herein reinforce the relevance of nutritional planning in order to achieve specific demands of tennis and maximize performance.

Keywords: tennis, diet, anthropometric profile

\section{INTRODUÇÃO}

A prática de exercícios físicos promove aumento do gasto energético, alterando, consequentemente, a necessidade energética ${ }^{(1)}$. Em atletas, a rotina de treinamento físico extenuante induz alterações consideráveis nas necessidades nutricionais. Portanto, a alimentação adequada, durante os períodos de treinamento e competição, é fundamental não somente para maximizar o desempenho(2), mas também para permitir a recuperação plena e a manutenção da saúde(3).
O tênis é uma atividade intermitente, que intercala movimentos explosivos de alta intensidade e curta duração com períodos de recuperação(4) ${ }^{(4)}$ Além disso, o tênis é o único esporte disputado em pisos diferentes e com bolas diferentes. A duração das partidas também varia bastante, de 60 até 300 minutos (melhor de três sets ou melhor de cinco sets) ${ }^{(4)}$. Essas características específicas sugerem que todos os sistemas energéticos são recrutados durante a prática desse esporte. Partindo desse pressuposto, a dieta desses atletas deveria preconizar 
o fornecimento adequado de todos os nutrientes. O carboidrato é uma importante fonte de energia, tanto nos picos anaeróbios, que dependem da degradação do glicogênio, quanto para a manutenção da glicemia em longo prazo ${ }^{(5)}$. A proteína exerce papel importante na manutenção da massa muscular, requisito fundamental para ações de força e potência, inerentes à prática desse esporte ${ }^{(5)}$. Já o aproveitamento metabólico adequado desses macronutrientes depende do aporte adequado de vitaminas e minerais, uma vez que os mesmos são cofatores em todos os processos de produção de energia e síntese de macromoléculas ${ }^{(5)}$. Logo, é inquestionável a relevância do planejamento alimentar de tenistas que visam o desempenho máximo.

As recomendações nutricionais dirigidas à população em geral não são suficientemente conclusivas em relação aos atletas ${ }^{(6-9)}$. Essa falta de consenso se deve, entre outras questões, às grandes especificidades de cada modalidade esportiva. Ademais, fatores como o nível de treinamento, idade, sexo, entre outros interferem nas necessidades nutricionais ${ }^{(10)}$.

É importante ressaltar que as variáveis antropométricas também podem influenciar o desempenho esportivo. Diversos estudos sugerem que as medidas antropométricas contribuem para o sucesso em determinada modalidade ${ }^{(11-14)}$. Para Ramana et al. ${ }^{(15)}$, o estudo sobre o perfil antropométrico de atletas tem como objetivo definir uma condição morfológica "ideal", específica de cada esporte. Além disso, a avaliação antropométrica pode ser considerada uma estratégia adicional para o monitoramento/controle do processo de treinamento.

Embora exista grande interesse sobre o consumo alimentar e o perfil antropométrico de atletas de diferentes modalidades, as informações sobre essas características em tenistas brasileiros são escassas. Além disso, é importante ressaltar que nenhum estudo prévio comparou o consumo alimentar e o perfil antropométrico de tenistas AM e PRO no Brasil. Partindo do pressuposto de que atletas PRO apresentam maior comprometimento com o esporte, o presente estudo levanta a hipótese de que jogadores AM e PRO poderiam apresentar perfil antropométrico e consumo alimentar diferenciados. Considerando essa lacuna do conhecimento, o objetivo deste estudo foi descrever aspectos relacionados com o consumo alimentar e o perfil antropométrico de atletas de tênis, comparando AM e PRO.

\section{MÉTODOS}

\section{Amostra}

Foram estudados 24 jogadores de tênis, do sexo masculino, com idade média de 18,0 \pm 1,4 anos. Esses atletas foram distribuídos em dois grupos: amadores ( $\mathrm{AM}=15$ tenistas posicionados no ranking da Confederação Brasileira de Tênis - CBT) e profissionais (PRO = nove tenistas com no mínimo um ponto na Associação dos Tenistas Profissionais - ATP). Todos os atletas estavam na fase final da pré-temporada para o ano de 2008 e treinavam diariamente em dois períodos, manhã e tarde, perfazendo o total diário de 3:30' em quadra e 2:30' em preparação física. Os tenistas avaliados concordaram em participar do presente estudo, de maneira voluntária, assinando o termo de consentimento livre e esclarecido. O procedimento experimental foi aprovado pela comissão de ética em pesquisa da Escola de Educação Física da Universidade de São Paulo (n 15/2008).

\section{Ingestão alimentar}

Apesar das conhecidas limitações ${ }^{(16)}$, o emprego de diários e recordatórios continua sendo a forma mais barata, prática e acessível para a avaliação do consumo alimentar ${ }^{(17)}$. Assim sendo, os jogadores foram instruídos a preencher três diários alimentares, em dias não consecutivos, incluindo dois dias úteis e um do final de semana. Os atletas deveriam relatar, de forma mais precisa possível, os alimentos e bebidas ingeridos durante as 24 horas do dia, em medidas caseiras. Os dados coletados pelos registros alimentares preenchidos foram calculados em energia (VET), macronutrientes e micronutrientes com auxilio do software Dietwin Professional 3.0. Os valores obtidos foram discutidos em relação ao valor estabelecido pelas atuais Dietary Reference Intakes - DRI's(18) e com os posicionamentos do American College of Sports Nutrition ${ }^{(6)}$, American Dietetic Association and Dietitians of Canadá(7) e da American Society of Sports Nutrition ${ }^{(9)}$.

\section{Avaliação antropométrica}

Para a avaliação do perfil antropométrico e da estimativa da composição corporal dos tenistas estudados, foram coletadas as seguintes medidas antropométricas, cujos procedimentos seguiram a padronização proposta por Lohman et al. ${ }^{(19)}$.

\section{Massa corporal total (MC) e estatura (E)}

A massa corporal total e a estatura foram determinadas utilizandose balança $\left(\right.$ Filizola $\left.^{\circledR}\right)$ tipo plataforma, com estadiômetro acoplado. Os atletas foram colocados no centro da plataforma, em posição ereta, em plano de Frankfurt, descalços, usando apenas sunga.

\section{Dobras cutâneas}

Para a aferição das dobras cutâneas foi utilizado compasso da marca Cescorf ${ }^{\circledR}$. As medidas foram realizadas no hemicorpo direito do indivíduo, sendo a marcação realizada a $1 \mathrm{~cm}$ de profundidade, após dois segundos do pinçamento ${ }^{(20)}$. $\mathrm{O}$ avaliado permaneceu em posição anatômica durante o procedimento ${ }^{(20)}$. Foram utilizados os seguintes pontos anatômicos: tricipital (TR), bicipital, subescapular (SB), suprailíaca (SI), peitoral (PE), abdominal (AB), axilar média (AM), coxa (CO), panturrilha medial (PM).

\section{Circunferências corporais}

A aferição das circunferências corporais foi realizada com fita métrica flexível de precisão de $0,5 \mathrm{~cm}$. As medidas foram realizadas com o avaliado em pé, estando a fita em ângulo reto em relação ao eixo do corpo $^{(20)}$. As regiões mensuradas foram: braquial (CBR), abdominal $(C A B)$, quadril $(C Q A)$, cintura (CCA) - (CCO), perna (CPE), torácica (CT).

\section{Estimativa da gordura corporal (GC)}

Para estimativa do percentual e conteúdo de gordura corporal foram utilizadas equações que empregam diferentes medidas de dobras cutâneas, tratando-se, portanto de modelos de dois compartimentos: Jackson et al..(21), Guedes ${ }^{(20)}$, Durnin \& Womersley ${ }^{(22)}$ e Petroski(23).

\section{Gasto energético}

O gasto energético diário dos atletas foi estimado a partir dos seguintes passos: a) Gasto energético basal: a partir das medidas de peso e estatura foi estimada a necessidade energética basal (GEB), de acordo com as propostas da Organização Mundial da Saúde (indivíduos entre 10 e 18 anos $=16,6 \times M C+77 \times E+572$; indivíduos acima de 18 anos $=15,4 \times M C$ $+27 \times E+717)^{(24)}$. b) Termogênese induzida pela dieta (TID): foi adotado o valor de 10\% do GEB(24). c) Gasto energético para a atividade física: foi estimado de duas diferentes maneiras, que foram posteriormente comparadas. No procedimento 1, foi utilizado fator de 2,1, que corresponde a atividades pesadas ${ }^{(24)}$. Já no procedimento 2, foi feita a transformação do gasto diário na atividade física durante os treinos de quadra e jogos em equivalentes metabólicos (METs), propostos por Ainsworth et al. (1).

\section{ANÁLISE DOS DADOS}

Os dados foram apresentados como média e desvio padrão. Após a confirmação da normalidade pelo teste de Kolmogorov-Smirnov, os grupos de atletas AM e PRO foram comparados a partir do teste $t$ para amostras independentes, adotando-se como significativo o valor de $p<0,05$. Para realização dos cálculos, foi utilizado o software SPSS versão 15.0. 


\section{RESULTADOS}

Não houve diferença na massa corporal e na estatura entre os tenistas AM e PRO (AM: 66,0 $\pm 5,0 \mathrm{~kg}$ e PRO: 69,5 $\pm 9,8 \mathrm{~kg} ; \mathrm{p}>0,05$ e AM: $175,6 \pm 2,7 \mathrm{~cm}$ e PRO: $177,9 \pm 4,3 \mathrm{~cm} ; \mathrm{p}>0,05$ ) (tabela 1). Também não houve diferença significativa entre os valores de dobras cutâneas e circunferências corporais em ambos os grupos.

Tabela 1. Variáveis antropométricas de tenistas amadores (AM) e profissionais (PRO).

\begin{tabular}{l|c|c}
\hline \multirow{2}{*}{ Variáveis antropométricas } & \multicolumn{2}{|c}{ Grupos } \\
\cline { 2 - 3 } & AM & PRO \\
\hline Estatura $(\mathrm{cm})$ & $175,6 \pm 2,7$ & $177,9 \pm 7,2$ \\
\hline Massa corporal $(\mathrm{kg})$ & $66,0 \pm 5,0$ & $69,5 \pm 9,8$ \\
\hline $\mathrm{IMC}\left(\mathrm{kg} / \mathrm{m}^{2}\right)$ & $22,5 \pm 0,8$ & $23,4 \pm 1,4$ \\
\hline
\end{tabular}

Resultados expressos em média \pm desvio padrão; AM ( $(n=15)$ e PRO ( $n=9)$.

A tabela 2 apresenta as estimativas do percentual de gordura corporal pelos diferentes protocolos. Não houve diferença significativa entre os grupos em nenhum dos protocolos adotados.

Tabela 2. Percentual de gordura corporal de tenistas amadores (AM) e profissionais (PRO).

\begin{tabular}{c|c|c|c|c}
\hline \multicolumn{1}{c|}{ Protocolo } & Grupo & \% GC & MG (kg) & MLG (kg) \\
\hline \multirow{2}{*}{ Jackson et al (1980)21 } & PRO & $9,0 \pm 4,6$ & $6,5 \pm 4,2$ & $62,9 \pm 7,1$ \\
\cline { 2 - 5 } & AM & $9,8 \pm 2,2$ & $6,5 \pm 1,8$ & $59,4 \pm 3,5$ \\
\hline \multirow{2}{*}{ Guedes (1998)20 } & PRO & $13,8 \pm 5,5$ & $9,3 \pm 5,1$ & $60,1 \pm 6,8$ \\
\cline { 2 - 5 } & AM & $13,7 \pm 2,4$ & $9,2 \pm 2,1$ & $56,8 \pm 3,1$ \\
\hline \multirow{2}{*}{$\begin{array}{l}\text { Durnin \& Womersley } \\
\text { (1974) }^{22}\end{array}$} & PRO & $14,2 \pm 4,2$ & $10,1 \pm 4,1$ & $59,3 \pm 7,2$ \\
\cline { 2 - 5 } & AM & $15,5 \pm 1,4$ & $10,6 \pm 1,0$ & $57,7 \pm 10,2$ \\
\hline \multirow{2}{*}{ Petroski (1995) ${ }^{23}$} & PRO & $12,3 \pm 4,2$ & $8,7 \pm 4,1$ & $60,7 \pm 7,3$ \\
\cline { 2 - 5 } & AM & $12,8 \pm 1,6$ & $8,5 \pm 1,5$ & $57,5 \pm 3,8$ \\
\hline
\end{tabular}

Resultados expressos em média \pm desvio padrão; $M G$ = massa gorda; $M L G=$ massa livre de gordura AM $(n=15)$ e PRO $(n=9)$.

A estimativa de gasto energético total (GET) foi semelhante entre AM e PRO para os dois procedimentos adotados (GEBa $\times 2,1^{\mathrm{a}}+\mathrm{TID}-\mathrm{AM}$ : $3.600 \pm 299 \mathrm{kcal}$ vs. PRO: $3.764 \pm 376 \mathrm{kcal})\left(\mathrm{GEB}^{\mathrm{a}}+\mathrm{MET}^{\prime} \mathrm{s}^{\mathrm{b}}+\mathrm{TID}-\mathrm{AM}:\right.$ $3.945 \pm 421 \mathrm{kcal}$ vs. PRO: $4.227 \pm 305 \mathrm{kcal}$ ) (tabela 3, p > 0,05)(a- OMS (24); $\mathrm{b}$ - Ainsworth et al.(1)). A ingestão energética total (VET) dos tenistas (AM: $2.730 \pm 702 \mathrm{kcal}$ e PRO: $3.212 \pm 782 \mathrm{kcal}$ ) é reportada na figura 1 ( $p>0,05)$. Em ambos os grupos, foi constatado o balanço energético negativo (figura 1).

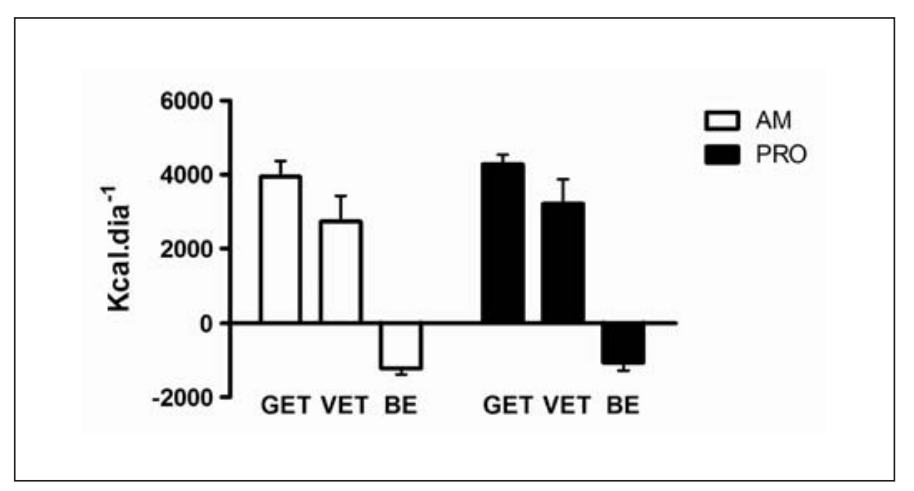

Figura 1. Comparação entre o relato de ingestão energética (VET) e o gasto energético total estimado (GET) de tenistas amadores $(A M ; n=15)$ e profissionais (PRO; $n=9)$. Resultados expressos em média \pm desvio padrão.
A tabela 3 apresenta a ingestão absoluta e relativa de macronutrientes e a tabela 4 descreve a ingestão de micronutrientes. Pode-se observar que a ingestão de carboidrato foi similar entre os grupos, atingindo o limite inferior da recomendação para atletas. Com relação aos micronutrientes, a ingestão de cálcio mostrou-se significativamente inferior ao estabelecido pelas DRI's $(p<0,05)$.

Tabela 3. Distribuição dos macronutrientes na dieta de tenistas amadores (AM) e profissionais (PRO).

\begin{tabular}{l|c|c|c|c|c|c}
\hline \multirow{2}{*}{ Nutriente } & \multicolumn{6}{c}{ Grupo } \\
\cline { 2 - 7 } & \multicolumn{3}{|c}{ AM } & \multicolumn{3}{c}{ PRO } \\
\hline \multirow{2}{*}{} & $\%$ & g/dia & g/kg/dia & $\%$ & g/dia & g/kg/dia \\
\hline Carboidratos & $61 \pm 6$ & $416,3 \pm 37,5$ & $6,3 \pm 0,5$ & $56 \pm 5$ & $449,7 \pm 40,5$ & $6,5 \pm 0,7$ \\
\hline Lipídios & $16 \pm 5$ & $48,7 \pm 14,5$ & $0,7 \pm 0,1$ & $24 \pm 4$ & $85,7 \pm 13,7$ & $1,2 \pm 0,2$ \\
\hline Proteínas & $23 \pm 4$ & $157,0 \pm 29,8$ & $2,4 \pm 0,2$ & $20 \pm 5$ & $160,6 \pm 40,2$ & $2,3 \pm 0,3$ \\
\hline
\end{tabular}

Resultados expressos em média \pm desvio padrão; AM $(n=15)$ e PRO $(n=9)$.

Tabela 4. Ingestão dietética de tenistas amadores (AM) e profissionais (PRO) em relação à ingestão recomendada de micronutrientes (DRI's).

\begin{tabular}{l|c|c|c}
\hline \multicolumn{1}{c|}{ Nutriente (mg) } & DRI's & AM & PRO \\
\hline Riboflavina (B2) & 1,3 & $1,6 \pm 0,9$ & $1,8 \pm 1,1$ \\
\hline Niacina (B3) & 16 & $26,2 \pm 18,9$ & $24,9 \pm 19,1$ \\
\hline Ac. pantotênico (B5) & 5 & $3,6 \pm 0,7$ & $3,4 \pm 0,9$ \\
\hline Acido ascórbico (C) & $75^{*} / 90^{* *}$ & $109,6 \pm 70,2$ & $113,1 \pm 83,9$ \\
\hline Vitamina E & 15 & $11,6 \pm 6,9$ & $11,1 \pm 8,9$ \\
\hline Cálcio (Ca) & $1.300^{*} / 1000^{* *}$ & $778,7 \pm 535,6^{a}$ & $782,6 \pm 491,9 a$ \\
\hline Ferro (Fe) & $11^{*} / 8^{* *}$ & $10,6 \pm 7,9$ & $11,2 \pm 9,3$ \\
\hline
\end{tabular}

Resultados expressos em média \pm desvio padrão; 1- DRI's * $=14-18$ anos;

** $=19-70$ anos. $a-p>0,05$ em relação a DRI's 14-18 anos.

\section{DISCUSSÃO}

Os principais achados do presente estudo foram: a) não foi observada diferença entre o perfil antropométrico de atletas AM e PRO; b) o consumo alimentar foi similar entre os atletas PRO e AM; c) foi detectado balanço energético negativo em ambos os grupos; e d) o consumo de cálcio foi inferior ao valor de referência (DRl's 14-18 anos) em ambos os grupos. Além disso, é importante ressaltar que o consumo de carboidratos atingiu o limite mínimo recomendado e a ingestão de proteínas foi superior aos valores sugeridos pela literatura.

O perfil antropométrico observado no presente estudo é muito semelhante ao resultado previamente reportado por Pereira ${ }^{(25)}$, que também avaliou o perfil antropométrico de tenistas brasileiros. A semelhança do perfil antropométrico entre os diferentes estudos pode ser explicada pelo fato de a composição corporal constituir um dos critérios para seleção dos indivíduos mais aptos. No presente estudo também foi observado que o perfil antropométrico de atletas AM e PRO é muito semelhante. Cabe ressaltar que os tenistas AM e PRO são submetidos a cargas de treinamento semelhantes. Além disso, mesmo em nível amador, esses atletas já passaram pelo processo de seleção imposto pela modalidade. Portanto, as cargas de treinamento similares e o processo de seleção podem estar relacionados com as semelhanças observadas entre os dois grupos de atletas.

Os resultados do percentual de gordura corporal estimados pelas fórmulas de Guedes ${ }^{(20)}$, Durnin \& Womersley ${ }^{(22)}$ e Petroski(23) são similares quando comparados com o estudo de Pereira ${ }^{(25)}$. Em outro estudo também conduzido em jovens tenistas brasileiros, Juzwiak et al.(27) 
reportaram que esse percentual reduzido pode ser vantajoso para o tênis, uma vez que os atletas devem executar movimentos explosivos (saques, smash, forehand, backhand e voleios) e desempenhar velocidade/agilidade na movimentação de quadra. Segundo Costa(26), o valor percentual de gordura corporal em atletas varia de 5 a 12\% nos homens, dependendo da modalidade esportiva.

Os resultados relacionados com o balanço energético no presente estudo (ingestão energética - gasto energético) sugerem a ocorrência de balanço energético negativo para os dois grupos de atletas. De acordo com a discussão estabelecida pela FAO/WHO/ONU(24), se os indivíduos se encontram, "na média em estado firme, com a composição corporal e o nível de atividade física apropriados", não se deve considerar o quadro indicativo de subconsumo. Portanto, a estabilidade do peso dos atletas, observada por mais de quatro meses antes do estudo, reforça a hipótese de que ocorreu sub-registro da ingestão alimentar. Provavelmente, essa discrepância entre o consumo e o gasto está associada ao sub-relato.

Scagliusi e Lancha Junior(28) reportam que o sub-relato é constituído pelo sub-registro e/ou subconsumo. O sub-registro é definido como o não relato de alimentos de fato consumidos e o subconsumo está relacionado com a diminuição do consumo alimentar causada pelo próprio instrumento de avaliação ou por outros fatores de causa fisiológica ou até patológica ${ }^{(29)}$. Ambos os processos envolvem componentes perceptivos, emotivos e cognitivos, ainda pouco explorados ${ }^{(30)}$. O comportamento do sub-relato é comum entre atletas ${ }^{(31-34)}$. No estudo de Edwards et al. ${ }^{(31)}$, utilizando o método da água duplamente marcada, foi observado que corredoras apresentam ingestão energética inferior (32\%) ao gasto energético. Hill \& Davies ${ }^{(32)}$, usando a mesma metodologia, também observaram significativo sub-relato em remadoras (em média -1.163kcal/dia). Em outro estudo, foi constatado que o sub-relato aumenta, progressivamente, durante a competição ${ }^{(33)}$. Nesse estudo, o sub-relato foi observado por três semanas, durante o Tour de France. Nesse período, o sub-relato foi de 13\% para primeira semana, 21\% para segunda e 35\% para a última semana. Os autores atribuíram o sub-relato ao comportamento de sub-registro, uma vez que o peso dos atletas não sofreu alteração ${ }^{(33)}$. Já no estudo de Fudge et al. ${ }^{(34)}$ também foi observado sub-relato por parte de corredores quenianos que reduzem, propositalmente, a sua ingestão calórica no período que antecede a competição. Entretanto, nesse último estudo ${ }^{(34)}$ o sub-relato foi, principalmente, associado ao subconsumo.

Alguns autores afirmam que os indivíduos tendem a relatar ingestões que sejam similares ao esperado pela população em geral ${ }^{(35)}$. Por exemplo, obesos tendem a reportar ingestão similar à de indivíduos não obesos; por sua vez, atletas tendem a reportar consumo semeIhante ao observado para indivíduos menos ativos ${ }^{(35)}$. Krebs-Smith et al. ${ }^{(36)}$ observaram sub-registro relacionado aos alimentos mais calóricos e com menor valor nutricional (bolos, tortas, salgadinhos, etc.), ou seja, alimentos considerados pouco saudáveis. O sub-registro seletivo pode ser consequência do maior nível de conhecimento e/ou preocupação com alimentação por indivíduos fisicamente ativos/atletas. Obviamente, é importante mencionar as limitações dos métodos empregados no presente estudo, tais como possíveis erros nos cálculos nutricionais da dieta, por limitações nas tabelas de alimentos disponíveis, ou ainda pela descrição de todos os ingredientes nas preparações culinárias. Além disso, uma vez que as necessidades energéticas foram estimadas por meio de equações desenvolvidas para a população não atleta, os dados reportados devem ser interpretados com cautela. Todavia, outros trabalhos que avaliaram a questão do balanço energético em atletas também evidenciaram a mesma magnitude de sub-relato que o presente estudo ${ }^{(31,32)}$.
Além do ajuste da ingestão energética ao gasto energético, é fundamental para melhor rendimento a adequação da qualidade e quantidade de macronutrientes. No que diz respeito aos carboidratos, já está bem estabelecido o papel desse macronutriente para o desempenho em atividades de longa duração, quando ingerido antes e durante o exercício de endurance ${ }^{(39,40)}$. O carboidrato parece ser ainda mais importante para atletas envolvidos em esportes intermitentes, nos quais o desgaste dos estoques de carboidratos (glicogênio muscular e hepático) é exacerbado ${ }^{(37,38)}$.

Considerando o posicionamento do ACSM/ADA/DC(6), a ingestão de carboidratos pelos atletas do presente estudo está adequada (nos limites mínimos sugeridos) tanto em termos percentuais, quanto em valores relativos à massa corporal. Os atletas relataram ingestão de carboidratos entre 56\%(PRO) a 61\%(AM) do total calórico consumido por dia. Isto significa, aproximadamente, $6 \mathrm{~g} / \mathrm{kg} / \mathrm{dia}$. O posicionamento estabelece que, na dieta diária, atletas devem ingerir valores relativos entre 6 e 10g/kg/dia. Entretanto, é importante ressaltar que os atletas estavam em fase final da pré-temporada. Em períodos competitivos, tanto o posicionamento ${ }^{(6)}$ quanto outros estudos ${ }^{(8)}$ têm demonstrado vantagens no aumento da ingestão desse nutriente, sendo sugerido o aumento para até $10 \mathrm{~g} / \mathrm{kg} / \mathrm{dia}$. É importante ressaltar que durante os torneios os tenistas jogam de três a quatro vezes por semana; consequentemente, durante a competição a reposição dos estoques de glicogênio poderá ser um fator limitante da performance nos últimos e mais importantes jogos ${ }^{(41)}$. Portanto, o planejamento nutricional desses atletas deveria priorizar estratégias para maximizar a ingestão de carboidrato, a fim de atender suas demandas do treinamento intenso e da rotina de competições. No tênis, os poucos trabalhos realizados sobre o efeito da ingestão/suplementação de carboidrato ainda apresentam resultados controversos $^{(41)}$, reforçando a necessidade de estudos adicionais.

Mesmo considerando a extrema importância dos carboidratos, é fundamental que não se desvie a atenção dos demais macronutrientes na dieta. Para que seja assegurada a ingestão de todos os nutrientes, a ingestão energética deve ser elevada, o que tipicamente ocorre entre atletas ${ }^{(6,7)}$.

As proteínas fornecem aminoácidos que, no caso de atletas, contribuem não somente para a manutenção da massa livre de gordura(42), mas também para respostas relativas à imunocompetência ${ }^{(43)}$. Embora possam ser oxidadas, as proteínas não desempenham papel significativo na geração de energia durante a atividade física intermitente de alta intensidade, a menos que a ingestão dos demais nutrientes energéticos esteja comprometida ${ }^{(44)}$. Muito se discute, sem um consenso, sobre a ingestão de proteínas por atletas, tanto em termos percentuais como em relação à massa corporal(45). Os atletas do presente estudo apresentaram tendência a ingerir percentuais elevados de proteínas na dieta. Juzwiak et al. ${ }^{(27)}$ reportam consumo excessivo de proteínas (75\% dos avaliados apresentaram consumo maior que 15\%) em jovens. No presente estudo, o consumo diário de proteínas também foi elevado (AM: $\sim 2,4 \mathrm{~g} / \mathrm{kg} /$ dia e PRO: $\sim 2,3 \mathrm{~g} / \mathrm{kg} / \mathrm{dia}$ ). O posicionamento do ACSM/ADA/DC ${ }^{(6)}$ sugere a ingestão de proteínas, para o exercício de endurance, de 1,2 a 1,4g/kg/dia, e para o exercício de força, de 1,6 a $1,7 \mathrm{~g} / \mathrm{kg} / \mathrm{dia}$. Já o posicionamento da International Society of Sports Nutrition $^{(9)}$ sugere intervalos entre 1,4 e 2,0 g/ $\mathrm{kg} / \mathrm{dia}$. As DRI's atuais ao abordar a questão da necessidade de proteínas concordam que esse nutriente é sujeito a grande variabilidade diária, e que episódios de consumo elevado não parecem ter efeitos colaterais significativos. Nesse contexto, as DRI's, admitindo a variabilidade da ingestão, sugerem como aceitável uma margem de ingestão entre 10 e 35\%(18).

Os lipídios, além de também participar de processos relacionados com a função imune, garantem a integridade de membranas celulares, em especial células do sistema nervoso ${ }^{(43,46)}$. O aumento da formação 
de espécies reativas e traumas durante exercícios de alta intensidade leva ao estado inflamatório, que pode ser atenuado, segundo alguns autores, através da ingestão de lipídios específicos como o EPA (ácido eicosapentanoico) e/ou DHA (ácido docosahexanoico)(47). Entretanto, não existem estudos na literatura sobre o papel dos lipídios na prática do tênis. No estudo conduzido por Juzwiak et al. ${ }^{(27)}$ foi observado que a maioria (80\%) dos tenistas avaliados consumia acima de 30\% do VET na forma de lipídios. No presente estudo, os grupos mantiveram o consumo desse macronutriente dentro dos limites recomendados ${ }^{(18,24)}$. Além disso, é importante ressaltar que não foi detectada diferença significativa com relação ao consumo de lipídios entre os grupos.

Embora, as vitaminas e os minerais não possam ser convertidos metabolicamente em energia, esses micronutrientes ajudam a regular os processos fisiológicos e bioquímicos do organismo, agindo como coenzimas, antioxidantes ${ }^{(48)}$ ou elementos de construção. No presente estudo, somente o cálcio apresentou desvio significativo em relação às DRI's em ambos os grupos ( $p>0,05$ em relação ao valor recomendado para 14-18 anos). O consumo adequado de cálcio é relevante em adolescentes, devido ao fato de os mesmos estarem em pico da formação de massa óssea ${ }^{(49)}$. Ainda, o metabolismo do cálcio sofre profundas modificações em resposta ao treinamento físico. Evans et al. ${ }^{(50)}$ identificaram elevação significativa dos marcadores bioquímicos do turnover de cálcio após quatro meses de treinamento físico, em homens e mulheres. Esse aumento comprova a importância da ingestão dietética desse nutriente para que sejam mantidos os processos de construção óssea, contração muscular e ainda a atividade de diversas enzimas dependentes de cálcio. Martin et al. ${ }^{(51)}$ constataram que as per-

\section{REFERÊNCIAS BIBLIOGRÁFICAS}

1. Ainsworth BE, Haskell WL, Leon AS, Jacobs DR, Montoye HJ, Sallis JF, et al. Compendium of physical activities: classification of energy costs of human physical activities. Med Sci Sports Exerc. 1993;25:71-80.

2. Probart CK, Bird PJ, Parker KA. Diet and athletic performance. Med Clin North Am. 1993;4:757-72.

3. Spriet LL, Peters SJ. Influence of diet on the metabolic responses to exercise. Proc Nutr Soc. 1998;57:25-33.

4. Fernandez J, Mendez-Villanueva A, Pluim BM. Intensity of tennis match play. Br J Sports Med. 2006;40:387-91.

5. Maughan RJ, Burke LM. Sports Nutrition. Malden, MA: Blackwell Science, 2002.

6. American College of Sports Medicine. Joint Position Statement: Nutrition and athletic performance. Med Sci Sports Exerc. 2000;32:2130-45.

7. American Dietetic Association na Dietitians of Canada. J Am Diet Assoc. 2000;12:1543-56.

8. Burke LM, Cox GR, Cummings NK, Desbrow B. Guidelines for daily carbohydrate intake: do athletes achieve them? Sports Med. 2001;31:267-99.

9. Campbell B, Kreider RB, Ziegenfuss T, La Bounty P, Roberts M, Burke D, et al. International Society of Sports Nutrition position stand: protein and exercise. J Int Soc Sports Nutr. 2007;4:8-13.

10. Hargreaves M. Exercise Metabolism. Champaing: Human Kinetics, 1995.

11. Barr SI, McCarger L, Crawford SM. Practical use of body composition analysis in sport. Sports Med. 1994;17:277-82.

12. Hoare DG. Predicting success in junior elite basketball players: the contribution of anthropometic and physiological attributes. J Sci Med Sport. 2000;3:391-405.

13. Bayios IA, Bergeles NK, Apostolidis NG, Noutsos KS, Koskolou MD. Anthropometric, body composition and somatotype differences of Greek elite female basketball, volleyball and handball players. J Sports Med Phys Fitness. 2006;46:271-80

14. Fernandes R, Barbosa T, Vilas-Boas JP. Fatores cineantropométricos determinantes em nataçăo pura desportiva. Rev Bras Cineantropom Des Hum. 2005;7:30-34

15. Ramana YV, Surya MVLK, Sudhakar SR, Balakrishna N. Effect of changes in body composition profile on VO2max and maximal work performance in athletes. JEPonline. 2004;7:34-9.

16. Trabulsi J, Schoeller DA. Evaluation of dietary assessment instruments against doubly labeled water, a biomarker of habitual energy intake. Am J Physiol Endocrinol Metab. 2001;281:E891-E999.

17. Rumpler WV, Kramer M, Rhodes DG, Moshfegh AJ, Paul DR. Identifying sources of reporting error using measured food intake. Eur J Clin Nutr. 2008;62:544-52.

18. Otten, JJ, Hellwig, J.P, Meyers, LD. Dietary reference intakes: The essential guide to nutrient requirements. Washington, DC: National Academy Press, 2006

19. Lohman TG, Roche AF, Martoll R. Anthropometric standardization reference manual. Champaign: Human Kinetic Books, 1991.

20. Guedes DP. Composição corporal: princípios, técnicas e aplicaçōes. Santa Catarina: Ceitec, 1998.

21. Jackson AS, Pollock ML, Ward A. Generalized equations for predicting body density of women. Med Sci Sports Exerc. 1980;12:175-82.

22. Durnin JV, Womersley J. Body fat assessed from body density and its estimation from skinfold thickness: measurements on 481 men and women aged from 16 to 72 years. Br J Nutr. 1974:32:77-97.

23. Petroski EL. Desenvolvimento e validaçăo de equações generalizadas para a estimativa da densidade corporal em adultos. [Tese de Doutorado - Programa de Pós-Graduaçăo]. Santa Maria (RS), Universidade Federal de Santa Maria, 1995.

24. FAO (Food and Agriculture Organization)/WHO (World Health Organization)/UNU (United Nations University), 1985. Necessidades de energia e proteína. Série de relatos técnicos 724. São Paulo: Roca, 1985

25. Pereira CF. Perfil corporal de tenistas participantes do campeonato brasileiro de tênis, ambos os sexos, categoria 16 anos: um relato cineantropométrico. Rev Trein Desp. 2001;4:33-9. das de cálcio pelo suor, em atletas do sexo feminino, eram diminuídas após a suplementação desse nutriente. Todavia, estudos adicionais são necessários para avaliar as consequências, em médio e longo prazo, da baixa ingestão de cálcio realizada por jovens tenistas.

\section{CONCLUSÕES}

Os resultados do presente estudo sugerem que o perfil antropométrico e o consumo alimentar de atletas amadores e profissionais são similares, refutando a hipótese inicial. Foi observado balanço energético negativo em ambos os grupos de atletas, possivelmente, explicado pelo sub-registro da ingestão alimentar. Estudos adicionais utilizando métodos mais fidedignos são necessários para avaliar o balanço energético no tênis. Com relação aos nutrientes, o consumo de carboidrato atingiu o limite mínimo e a ingestão de proteína superou os valores máximos sugeridos pela literatura. Também merece destaque a baixa ingestão de cálcio realizada pelos tenistas. Os resultados do presente estudo reforçam a importância do planejamento alimentar voltado para atletas, a fim de adequar a dieta e maximizar o desempenho esportivo.

\section{AGRADECIMENTOS}

Rodrigo Vitasovic Gomes agradece à Capes pela bolsa de mestrado. Também agradecemos o apoio do CNPq (Projeto 563967/2008-0).

Todos os autores declararam não haver qualquer potencial conflito de interesses referente a este artigo.
26. Costa RF. Composição corporal: teoria e prática da avaliaçăo. São Paulo: Manole, 2001.

27. Juzwiak CA, Amâncio OS, Vitalle MSS, Pinheiro MM, Szejnfeld VL. Body composition and nutritional profile of male adolescent tennis players. J Sports Sci. 2008;26:1209-17.

28. Scagliusi FB, Lancha Junior AH. Subnotificação da ingestão energética na avaliaçăo do consumo alimentar. Rev Nutr. 2003;16:471-81.

29. Goris AHC, Westerterp-Plantenga MS, Westerterp KR. Undereating and underrecording of habitual food intake in obese men: selective underreporting of fat intake. Am J Clin Nutr. 2000;71:130-4.

30. Krestch MJK, Fong AKH, Green MW. Behavioral and body sizes correlates of energy intake underreporting by obese and normal-weight women. J Am Diet Assoc. 1999;99:300-6.

31. Edwards JE, Lindeman AK, Mikesky AE, Stager JM. Energy balance in highly trained female endurance runners. Med Sci Sports Exerc. 1993;25:1398-404.

32. Hill RJ, Davies PS. Energy intake and energy expenditure in elite lightweight female rowers. Med Sci Sports Exerc. 2002;34:1823-9.

33. Westerterp KR, Saris WH, Van Es M, Ten Hoor F. Use of the doubly labeled water technique in humans during heavy sustained exercise. J Appl Physiol. 1986;61:2162-7.

34. Fudge BW, Westerterp KR, Kiplamai FK, Onywera VO, Boit MK, Kayser B, et al. Evidence of negative energy balance using doubly labelled water in elite Kenyan endurance runners prior to competition. Br I Nutr. 2006;95:59-66.

35. Burke LM. Energy needs of Athletes. Can J Appl Physiol. 1998;8:160-74.

36. Krebs-Smith SM, Graubard BI, Kahle LL, Subar AF, Cleveland LE, Ballard-Barbash, R. Low energy reporters vs. other reporters: a comparison of reported food intake. Eur J Clin Nutr. 2000;54:281-7.

37. Costill DL, Miller JM. Nutrition for endurance sport: carbohydrate and fluid balance. Int J Sports Med. 1980;1:2-14.

38. Coyle EF. Carbohydrate feeding during exercise. Int J Sports Med. 1992;13:S126-S8

39. Alhborg B, Bergstrom J, Ekelund LG, Hultman E. Muscle glycogen and muscle electrolytes during prolonged physical exercise. Acta Phys Scand. 1967;70:129-42.

40. Bergstrom J, Hermansen L, Hultman E, Saltin B. Diet, muscle glycogen and physical performance. Acta Phys Scand. 1967;71:140-50.

41. Kovacs MS. Carbohydrate intake and tennis: are there benefits? Br J Sports Med. 2006;40:1-5.

42. Layman DK. The role of leucine in weight loss diets and glucose homeostasis. J Nutr. 2003;133:2615-7S.

43. Nieman DC. Immunonutrition support for athletes. Nutr Rev. 2008;66: 310-20.

44. Lambert CP, Frank LL, Evans WJ. Macronutrient considerations for the sport of bodybuilding. Sports Med. 2004;34:317-27.

45. Lemon PWR. Beyond the zone: protein needs of active individuals. J Am Coll Nutr. 2000;19:513S-21S.

46. Innis SM. Dietary (n-3) fatty acids and brain development. J Nutr. 2007;137:855-9.

47. Simopoulos AP. Omega-3 fatty acids and athletics. Curr Sports Med Rep. 2007;6:230-6.

48. Duntas LH. Oxidants, antioxidants in physical exercise an relation to thyroid function. Horm Metab Res. 2005;37:572-6.

49. Calbet JAL, Moysi JS, Rodriguez LP. Bone mineral content in professional tennis players. Cal Tissue Int. 1998;62:491-6.

50. Evans RK, Antczak AJ, Lester M, Yanovich R, Israeli E, Moran DS. Effects of a 4-month recruit training program on markers of bone metabolism. Med Sci Sports Exerc. 2008;40:S660-S70.

51. Martin BR, Davis S, Campbell WW, Weaver CM. Exercise and calcium supplementation: effects on calcium homeostasis in sportswomen. Med Sci Sports Exerc. 2007;39:1481-6. 